\title{
On Bio-Deterioration of Solar Reflective Materials: An Innovative Experimental Procedure to Accelerate the Ageing Process of Surfaces
}

\author{
Giulia Santunione $^{1}$, Chiara Ferrari ${ }^{1}$, Alberto Muscio ${ }^{1}$ and Elisabetta Sgarbi ${ }^{2}$ \\ ${ }^{1}$ Department of Engineering “Enzo Ferrari”, University of Modena and Reggio Emilia, Italy, \\ giulia.santunione@unimore.it \\ ${ }^{2}$ Department of Life Science, University of Modena and Reggio Emilia, Italy, \\ elisabetta.sgarbi@unimore.it
}

\begin{abstract}
Solar reflective materials are one of the most efficient solution to the urban heat island effect, according to which temperatures, in urban areas are significantly higher than those in the surrounding rural areas. Solar reflective materials (SRM) are characterized by the ability to reflect solar radiation in the whole wavelength spectrum remaining cooler under the sun. The importance of radiative properties focused the attention more on SRM subjected to aging processes instead of the new products. According to this, several standards are, nowadays, available to investigate natural (ASTM G7, ANSI CRRC/S100) and accelerated (ASTM D7897) aging. Unfortunately, these standards do not take into account the presence and the growth of microorganisms such as algae and cyanobacteria, which can be responsible not only for the decrease of radiative properties against time, but also for the decay of physical and chemical properties of SRM themselves. This study outlines an experimental procedure to accelerate natural bio-deterioration of building materials through a laboratory test. Two types of SRM have been involved, studying their thermal properties before and after the bio-deterioration accelerated treatment. The methodology involves the use of a Temporary Immersion System (TIS bioreactor), which is based on the temporized soaking of materials samples with a liquid growth medium inoculated by selected species of algae or cyanobacteria. The system promotes a homogeneous contact between the materials surfaces and these microorganisms blowing sterilized air into the medium inside the bioreactor chamber. Within three weeks into TIS bioreactor, materials samples have shown large areas of biofilm deposition on themselves. The experimental procedure, hence, confirms the capability of the system to optimize environment conditions where the growth of microorganism and their interactions with materials results accelerated. Very high attention is given to the aim to make the procedure repeatable. For this proposal, all the variable factors are minimized, maintaining constant the environmental condition and working on sterility in order to do not contaminate or alter the growth inside the bioreactor.
\end{abstract}

Keywords: Solar Reflectance, Colorimetry, Bio-Deterioration, Bioreactor.

\section{Introduction}

Building materials are affected by several aesthetical and functional issues due to ageing processes which can be related to physical, chemical or biological factors (Sleiman et al. 2014). Among these factors, the biological growth represents a phenomenon in addition to physical and chemical problems due to weathering and soiling, which accelerates the fouling process of outdoor materials (Ferrari et al. 2015). The biofilm changes the aesthetical appearance of materials and can even compromise the durability of structures by corrosion and biodeterioration induced by the microorganisms (Maury-Ramirez et al., 2013). 
Several studies on natural conditions suggests that bio colonization is primarily related to the physical characteristics of the stone surface (porosity, roughness, and permeability) and secondarily to the nature of substratum (Gaylarde et al., 2011). Microorganisms produce variously coloured patinas on the materials inducing aesthetical damage (Ortega-Calvo et al., 1995). Biological growth also exerts a pressure inside the pores on the surface, resulting in biogeophysical deterioration, i.e. the surface detachment and superficial losses. Definitively, microorganisms play an important role in the deterioration of building materials, being responsible for aesthetic, biogeophysical and biogeochemical damage.

Considering Solar Reflective Materials (SRM), bio-deterioration process takes part to important performances losses in terms of surface thermal properties, such as solar reflectance and thermal emittance. Since studying microbial growth under natural conditions is too slow as an investigation approach to develop and to improve industrial products, several studies have been carried out on surface bio-ageing with the aim of analyse and to assess the energy impact on the buildings (Cheng et al., 2011; Cheng et al.,2012; Gaylarde and Gaylarde, 2005; Mastrapostoli et al., 2016). However, due to its the wide variability depending on environmental conditions and mainly by geographical position of the building, bio-deterioration is not yet being completely understood and quantify by the scientific community.

Thus, this study is aimed to study biological colonization on building materials in a repeatable and reproducible way, in order to quantify the changes on the surfaces properties due to biological growth. An innovative and laboratory protocol to accelerate biological colonization effects on building surfaces by different pioneering organisms (algae and cyanobacteria) is here presented. The goal was to provide a faster, reproducible and reliable test method to assess the long term behaviour of building surfaces relevant to, amon
energy performance for cooling. 2 Material and Methods

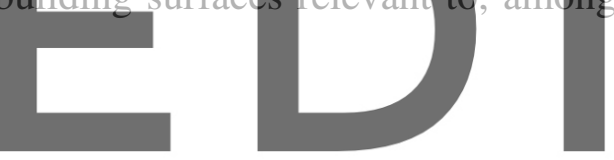
other,

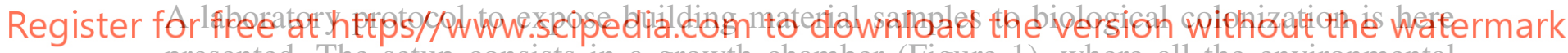
presented. The setup consists in a growth chamber (Figure 1), where all the environmental factors have been maintained constantly monitored and stable. The device chosen as growth chamber is a TIS (Temporary Immersion System) bioreactor (Welander et al., 2014).

a.

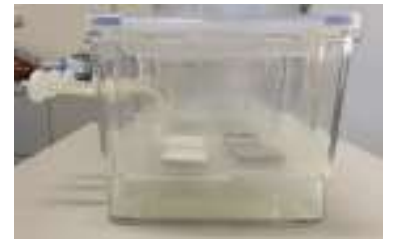

b.

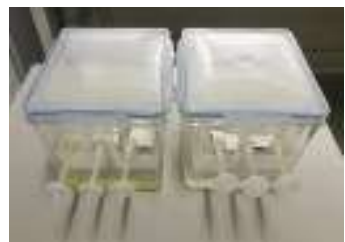

Figure 1. (a) TIS bioreactor equipped with the samples; (b) couple of TIS bioreactors for different environmental microorganisms (Chlorella mirabilis and Nostoc commune), start time of the test (t0).

It is a sealing growth camera where the samples are placed on a tray and temporarily flooded by $500 \mathrm{ml}$ of culture medium, inoculated by selected microorganisms according to 1:50 concentration rate. The submersion is induced by a pump $(10 \mathrm{~W})$, which inputs sterilized air inside the liquid medium in order to raise the level of that and submerge the samples on the tray. The level of the medium is subsequently lowered by stopping the air supply into the 
medium and raising the air pressure inside the camera, through a second pump $(5 \mathrm{~W})$. These working conditions were turned on and off trough a complete automated system. A complete bio-ageing cycle lasts 3 weeks and two types of building material were involved in this preliminary work: single ply cool white membrane (WM) and asphalt shingle (AS). Two samples for each type of material were included for each bioreactor, and each bio-ageing cycle was performed in triplicate in order to get statistically significant results.

Two specie of different microorganisms were used separately to study biofilm formation on sample surfaces: the green microalga Chlorella mirabilis and the cyanobacteria Nostoc commune. They were chosen among the huge variability of environmental ubiquitous organisms (Ferrari et al. 2015) and different culture medium were used for each microorganism: Bold Basal Medium (BBM) (ref) was applied for Chlorella m. and Blue Green medium (BG11) for Nostoc $c$. TIS bioreactors were put into the incubator (Biolog, AG-System) where the temperature and relative humidity level was stable $\left(23^{\circ} \mathrm{C}\right.$ and $50 \%$ respectively), and $14 \mathrm{~h}$ light/10h dark periods are contemporarily alternated by a fluorescent lamp system.

Since the study is finalized to develop a repeatable and reproducible protocol for bio-ageing trial, TIS bioreactors and culture medium are therefore completely sterilized before use to minimize the variability of environmental factors and avoid contamination of the biological cultures on growth. Two different sample submersion times were investigated: 10 minutes/6 times a day every 4 hours (setup \#1) and 30 minute /6 times a day every 4 hours (setup \#2): each time condition has been tested three repeated times as abovementioned. The complete

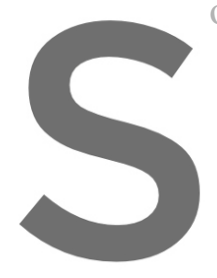
overview on experime
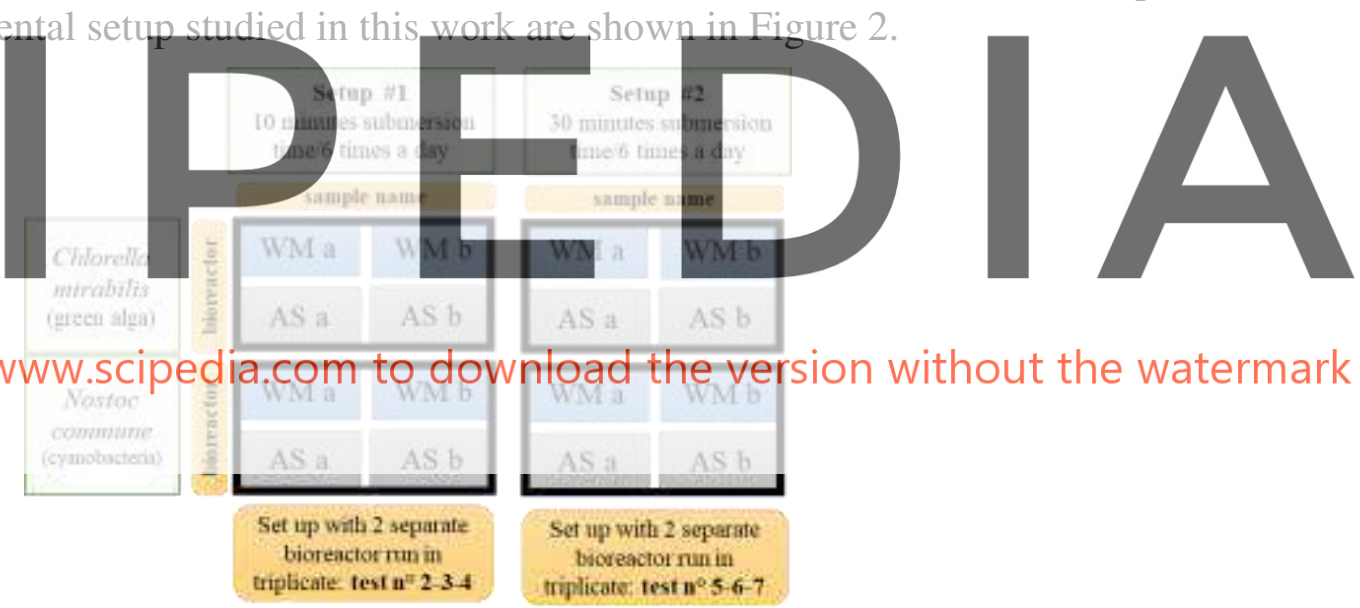

Register for free at https//www.scipedia.com to downioad the version without the watermark
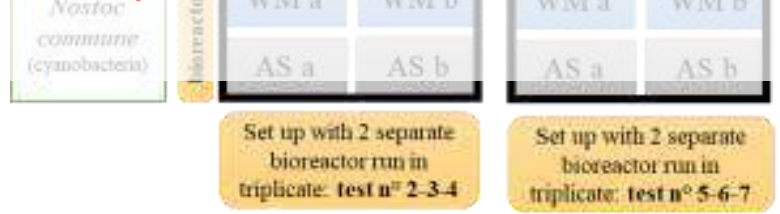

Figure 2. Bio-ageing experimental design: 2 bioreactors types were prepared for each trial; 4 samples were included into each bioreactor (double samples for each material); two setups were studied; the trials have been run in triplicate.

Such "flood and dry" approach tries to replicate the natural wetting and dry cycles that typically occur outdoor on the building surfaces. Reproducing a bio-deterioration phenomenon in laboratory allows to choose and set up stable environmental conditions, which are the optimum for biological growth, and to compress colonization time. 


\subsection{Material Characterization}

Material characterization was performed on sample surface before and after the bio-ageing test through several analyses. Firstly, surface microstructure were investigated by Environmental Scanning Electron Microscope (ESEM, Quanta200) operating in low vacuum conditions; Solar Reflectance $\left(\rho_{\text {sol }}\right)$ was analyzed by UV-Vis-Nir Spectrophotometer (Jasco V-670), according with ASTM Standard E903 (ASTM E903, 1996). To evaluate how the surface color was modified during the whole campaign $\mathrm{L}^{*} \mathrm{a} * \mathrm{~b} *$ values were calculated both on new and aged samples starting from solar reflectance values in visible region $(\lambda$ range: $300-800 \mathrm{~nm}) . \Delta \mathrm{E}$ values were calculated by means of $\Delta \mathrm{E}=\sqrt{\left(\mathrm{L}_{1}^{*}-\mathrm{L}_{2}^{*}\right)^{2}+\left(\mathrm{a}_{1}^{*}-\mathrm{a}_{2}^{*}\right)^{2}+\left(\mathrm{b}_{1}^{*}-\mathrm{b}_{2}^{*}\right)^{2}}$ to quantify the difference in color between the two stages of aging. Statistical analysis by ANOVA (Past3 Software) has been run in order to evaluate the repeatability of the preliminary protocol.

\section{Results and Discussion}

The differences collected between setup\#1 and setup \#2 trials are visibly high as the example reported in Figure 3. The first set up trial, reported in triplicate, shows a weaker but more homogeneous growth on materials samples then the second set up trials, where the submersion time has been 30 minutes for 6 times a day.

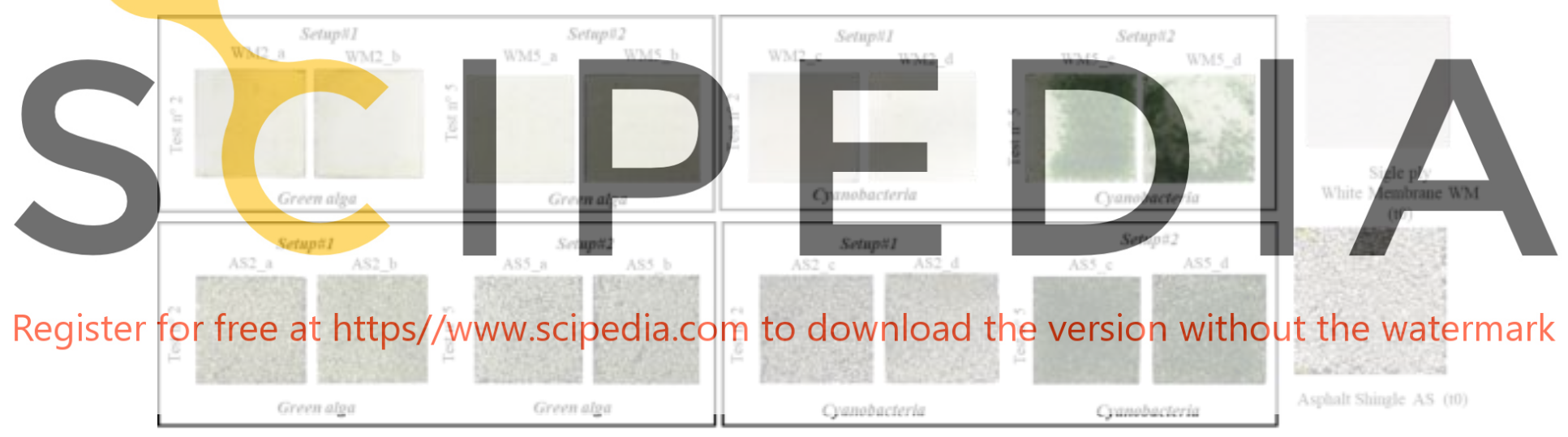

Figure 3. WM and AS aged samples are shown: $a$ and $b$ samples come from bioreactor run with green alga inoculum; $c$ and $d$ ones come from bioreactor run with cyanobacteria inoculum; the results from 1 (test $\mathrm{n}^{\circ} 2$ and test $n^{\circ} 5$ ) of 3 repeated tests by both setups types are shown; on the right, new (t0) sample are shown.

\subsection{Electron Microscopy}

Micromorphology and chemical composition analysis through Electron Microscopy carried out on samples before colonization, additional analysis were performed on aged samples immediately after the end of the bio-ageing trials. This allow to check microorganisms while they were still alive. Figure 4 shows the two different colonies of organisms in separate TIS bioreactor on AS sample: green alga (Chlorella m.) build spherical shape colonies (Fig. 4a) and cyanobacteria (Nostoc c .) are set in filamentous colonies (Fig. 4b).

Roughness on AS samples is high due to the presence of grains: this create the presence of several niches on surfaces, which represents a suitable place for biological growth thanks the 
longer staying of humidity. $\mathrm{C}$ is the most aboundant element in both AS $(65.0 \% \pm 22.7 \%)$ and $\mathrm{WM}(96.6 \% \pm 4.4 \%)$ materials, but AS samples are richer in elemental diversity: traces of $\mathrm{Mg}$, $\mathrm{Al}, \mathrm{Si}, \mathrm{S}, \mathrm{K}, \mathrm{Ca}, \mathrm{Ti}, \mathrm{Fe}$ compounds have been detected by ESEM.

a.

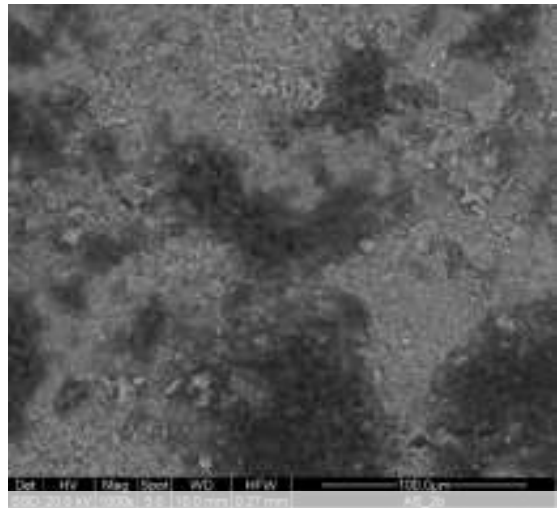

b.

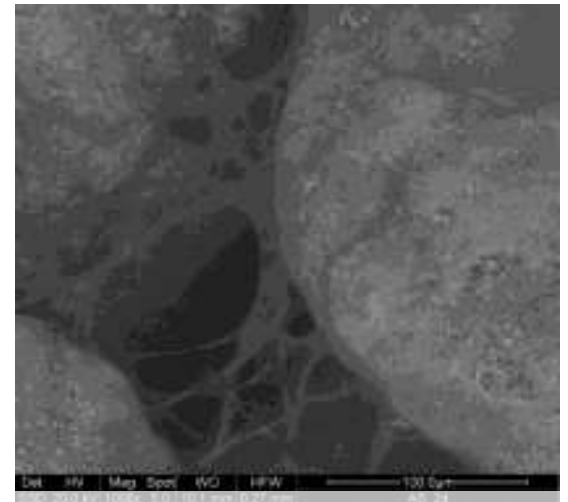

Figure 4. ESEM images from ESEM analysis: (a) Chlorella $m$. spherical cells colonies on AS sample; (b) Nostoc c. filamentous structure on AS sample. Mag. 1000X.

\subsection{Solar Reflectance}

Solar reflectance $\left(\rho_{\text {sol }}\right)$ values and standard deviation calculated on average values between samples exposed to the same microorganism ( $a$ and $b$ to Chlorella m. and $c$ and $d$ to Nostoc c.) have been reported in Table 1 . New and aged values are shown. Results have been elaborated comparing the same material $\rho_{\text {sol }}$ valyes between two different seturp trigls (\#1 and \#2) a within the same set

microorganisms (green Solar reflectance submersion time (setup \#1 and \#2) highlight E D. rising the strong variability among setups. Moreover, the colonization level due to green alga

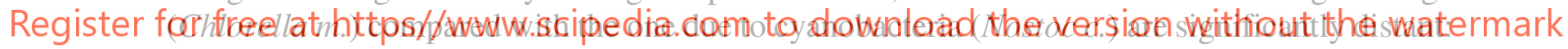
they have been evaluated separately.

Table 1 shows psoi decrease considering only $a$ and $b$ samples, before and after bio-ageing process. Samples $a$ and $b$, exposed to green alga, underline a lower $\rho_{\text {sol }}$ decrease through setup \#2 compared with setup \#1. This is true for both WM and AS samples. A reverse situation was achieved on samples $c$ and $d$ for both material types, exposed to cyanobacteria: the $\rho_{\text {sol }}$ decrease is higher through setup \#2, where the submersion time is tripled compared with setup \#1.

Statistical analysis among solar reflectance results obtained through repeated trials within the same setup type was performed. TIS bioreactor system, in particularly the case with green alga and setup\# 1 shows a good repeatability: the differences among $\rho_{\text {sol }}$ on the same material type (AS or WM) from different trials are not significant ( $\mathrm{p}$-value $>0.05$ ). However, this not occurs on $\rho_{\text {sol }}$ measured on aged samples $c$ and $d$ exposed to cyanobacteria colonization, neither for setup\#1 and setup \#2: in these cases, the statistical analysis highlight high significant differences among repeated trials ( $\mathrm{p}$-value $<<0.05)$. 
Table 1. Solar reflectance values by ASTM E903 are shown: AS and WM samples from both bioreactors with green alga $(a-b)$ and cyanobacteria $(c-d)$ inoculum; mean values on $a-b$ and $c-d$ samples are reported; $\Delta \rho_{\text {sol }}$ between new and aged samples are reported; SD among repeated $\rho$ sol measurements are considered.

\begin{tabular}{|c|c|c|c|c|}
\hline $\begin{array}{c}\text { Sample } \\
\text { name }\end{array}$ & $\begin{array}{c}\rho_{\text {sol }} \\
\text { (new) }\end{array}$ & $\begin{array}{c}\text { Mean } \rho_{\text {sol }} \\
(\text { aged) }\end{array}$ & $\Delta \rho_{\text {sol }}$ & SD \\
\hline AS2 $2 a-b$ & 0.304 & 0.260 & -0.045 & 0.011 \\
\hline $\mathrm{AS} 3 \_a-b$ & 0.304 & 0.275 & -0.029 & 0.021 \\
\hline AS4_a- $b$ & 0.303 & 0.229 & -0.071 & 0.030 \\
\hline AS5_a- $b$ & 0.304 & 0.294 & -0.010 & 0.014 \\
\hline AS6 $6 \_a-b$ & 0.304 & 0.295 & -0.008 & 0.005 \\
\hline AS7_a- $b$ & 0.308 & 0.312 & 0.004 & 0.010 \\
\hline
\end{tabular}

\begin{tabular}{ccccc}
\hline $\begin{array}{c}\text { Sample } \\
\text { name }\end{array}$ & $\begin{array}{c}\rho_{\text {sol }} \\
\text { (new) }\end{array}$ & $\begin{array}{c}\text { Mean } \rho_{\text {sol }} \\
\text { (aged) }\end{array}$ & $\Delta \rho_{\text {sol }}$ & SD \\
\hline AS2_c-d & 0.305 & 0.281 & -0.024 & 0.012 \\
\hline \multirow{2}{*}{ AS3_c-d } & 0.305 & 0.280 & -0.025 & 0.002 \\
\hline AS4_c-d & 0.298 & 0.232 & -0.066 & 0.015 \\
\hline \multirow{2}{\#}{ AS5_c-d } & 0.303 & 0.218 & -0.085 & 0.007 \\
\hline \multirow{2}{\#}{ AS6_c-d } & 0.306 & 0.203 & -0.103 & 0.005 \\
\hline AS7_c-d & 0.293 & 0.249 & -0.044 & 0.008 \\
\hline
\end{tabular}

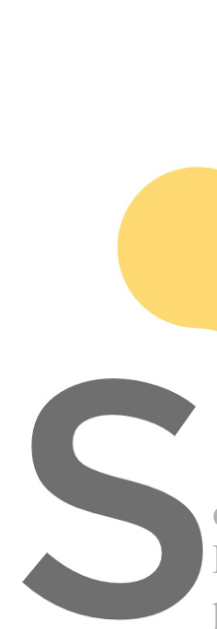

\begin{tabular}{|c|c|c|c|c|}
\hline Sample name & $\begin{array}{c}\rho_{\text {sol }} \\
\text { (new) }\end{array}$ & $\begin{array}{l}\text { Mean } \rho_{\text {sol }} \\
\text { (aged) }\end{array}$ & $\Delta \rho_{\text {sol }}$ & SD \\
\hline WM2_a- $b$ & 0.837 & 0.801 & -0.037 & 0.002 \\
\hline WM3 $a-b$ & 0.837 & 0.759 & -0.078 & 0.003 \\
\hline WM4_a-b & 0.837 & 0.804 & -0.033 & 0.007 \\
\hline WM5_ $a-b$ & 0.836 & 0.760 & -0.076 & 0.007 \\
\hline WM6_a-b & 0.827 & 0.769 & -0.058 & 0.012 \\
\hline WM7_a-b & 0.822 & 0.787 & & 0.018 \\
\hline
\end{tabular}

\begin{tabular}{ccccc}
\hline $\begin{array}{c}\text { Sample } \\
\text { name }\end{array}$ & $\begin{array}{c}\rho_{\text {sol }} \\
\text { (new) }\end{array}$ & $\begin{array}{c}\text { Mean } \rho_{\text {sol }} \\
\text { (aged) }\end{array}$ & $\Delta \rho_{\text {sol }}$ & SD \\
\hline WM2_c-d & 0.837 & 0.788 & -0.049 & 0.005 \\
\hline WM3_c-d & 0.837 & 0.753 & -0.084 & 0.004 \\
\hline WM4_c-d & 0.837 & 0.775 & -0.061 & 0.013 \\
\hline WM5_c-d & 0.832 & 0.600 & -0.232 & 0.037 \\
\hline WM6_c-d & 0.828 & 0.705 & -0.123 & 0.024 \\
\hline WM7_c-d & 0.819 & 0.574 & -0.244 & 0.034 \\
\hline underlines how the main difference \\
\hline reflectance spectrum, as showed in \\
\hline better evaluate the results of bio-ageing
\end{tabular}

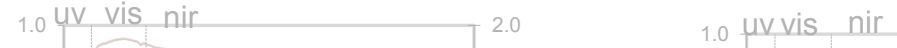

Register for free af. https//www.scipedia.com to download the version without the watermark

a.

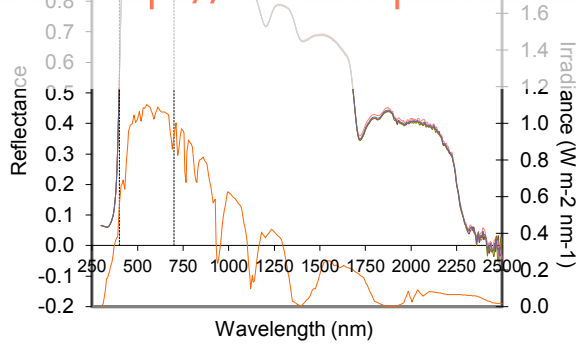

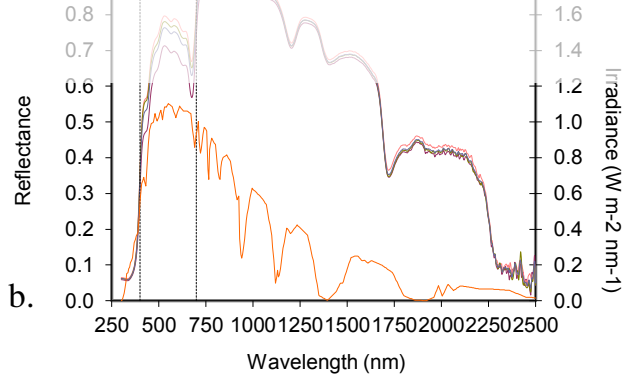

Wavelength (nm)

Figure 5. ASTM Solar Reflectance spectral chart on WM sample: (a) results on new sample; (b) results on bioaged sample. 3 repeated measurements on the same sample have been performed.

\subsection{Colorimetry}

Figure 5 reports the colorimetric differences between new ( $\mathrm{L}^{*}$ values) and aged $\left(\Delta \mathrm{L}^{*}\right.$ and $\left.\Delta \mathrm{E}\right)$ material types. Colorimetric differences have been calculated using the difference $\rho_{\text {sol }}$ within 300-800-nm $\lambda$ range (visible region). $\Delta \mathrm{L}^{*}$ and $\Delta \mathrm{E}$ have been assessed comparing all the samples $(a, b, c, d)$ coming from two different setup: again, the repeatability was described within the three trials with the same setup. These results confirm what noticed through solar reflectance: 
while the colonization level and solar reflectance decrease showed a good repeatability within the trials of setup\#1, this was not happened to setup \#2, where sample submersion time was tripled compared with \#1.
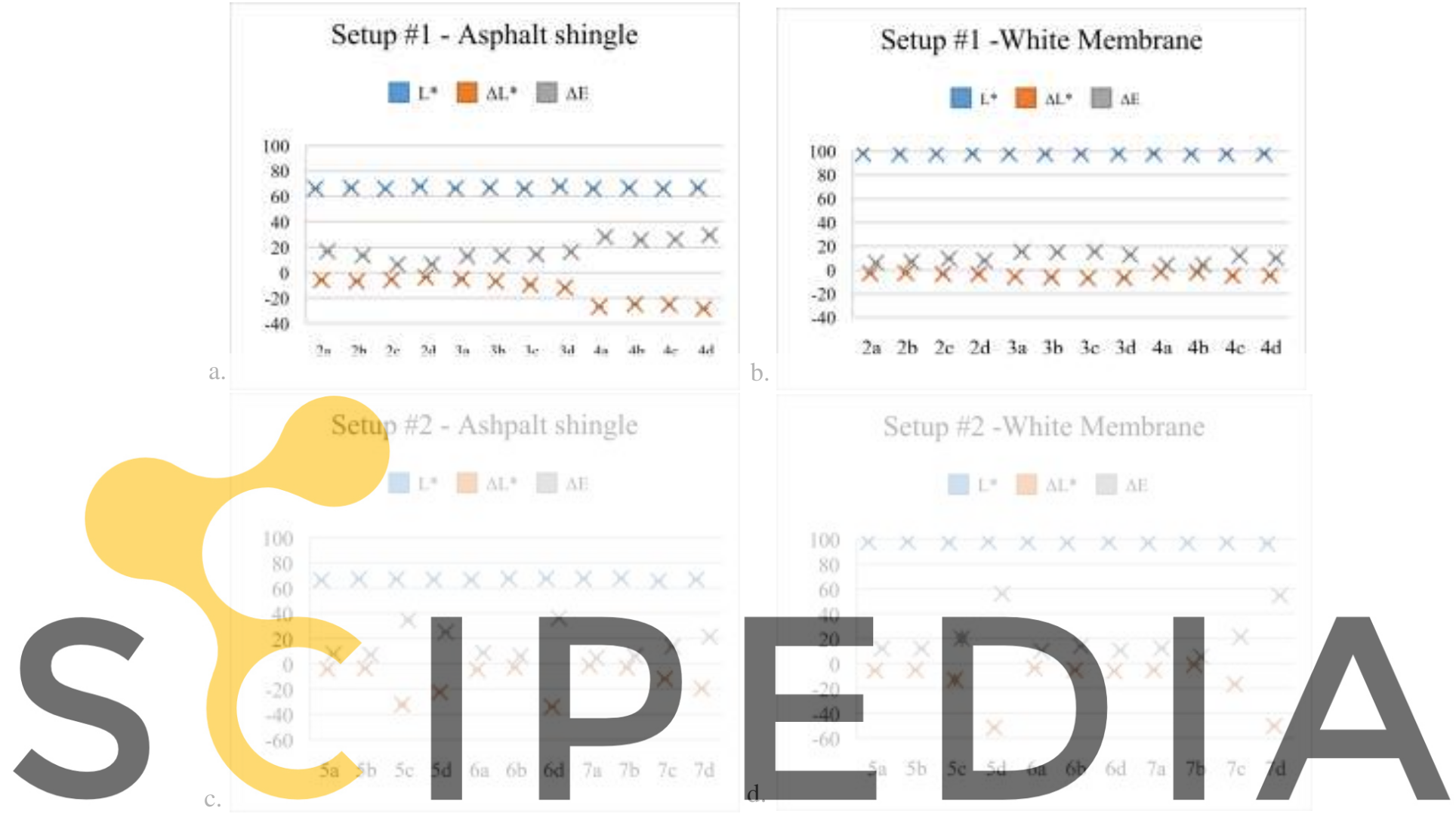

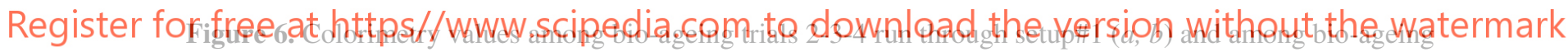
trials 5-6-7 run through setup\#2 $(c, d)$. All sample values are shown.

Microorganisms pigments are the main responsible of aesthetical damage on surfaces and they sharply contribute to losses in solar reflectance, as observed in this study. As shown in Figure $5, \Delta \mathrm{L}^{*}$ and $\Delta \mathrm{E}$ are averagely higher on $\mathrm{AS}$ samples (mean $\Delta \mathrm{L}^{*}-13.4 \pm 1.3$; mean $\Delta \mathrm{E}$ $17.5 \pm 1.1$ ) than in $\mathrm{WM}$ ones (mean $\Delta \mathrm{L}^{*}-4.3 \pm 0.5$; mean $\Delta \mathrm{E} 9.9 \pm 0.9$ ) for setup $\# 1 . \Delta \mathrm{L}^{*}$ and $\Delta \mathrm{E}$ from setup \#2 are similar to \#1 for AS samples (mean $\Delta \mathrm{L}^{*}-14.4 \pm 1.5$; mean $\Delta \mathrm{E} 17.2 \pm 1.3$ ), but the colorimetric distances from new and aged surfaces are deeper.

\section{Conclusions}

This work provides a preliminary laboratory bio-ageing protocol suitable to study the biodeterioration dynamics on building materials surfaces, in particular solar reflective materials. Since biological growth variability that occurs on surfaces in natural environment is really high, the bio-ageing protocol here proposed has been thought in order to keep stable and monitored all the laboratory environmental factors. Two specie of microorganisms, Chlorella $\mathrm{m}$. and Nostoc c., and two different laboratory setup have been investigated (setup\#1 and setup\#2) in 
order to study the more repeatable experimental conditions. Solar reflectance and colorimetry analysis on repeated trials shows setup\#1 (where 10 minutes of sample submersion time are repeated 6 times a day every 4 hours) as the most repeatable, and specifically when run with the green alga (Chlorella $\mathrm{m}$.). All the other experimental conditions achieved differences among the same sample type statistically significant. Even though the challenge to make repeatable something that it is not for its meaning definition, the preliminary bio-ageing setup\# 1 confirms the capability of the system to optimize environment conditions where the growth of microorganisms and their interactions with materials results faster, providing a way to investigate bio-deterioration processes on materials.

\section{ORCID}

Giulia Santunione http://orcid.org/0000-0001-6133-108X

Chiara Ferrari http://orcid.org/0000-0003-2188-6464

Alberto Muscio http://orcid.org/0000-0002-3085-3958

Elisabetta Sgarbi http://orcid.org/0000-0003-4366-6956

\section{References}

Cheng, Meng-Dawn D., Susan M. Pfiffner, William A. Miller and Paul Berdahl. (2011). "Chemical and Microbial Effects of Atmospheric Particles on the Performance of Steep-Slope Roofing Materials." Building and Environment 46(5):999-1010.

Cheng, Meng Dawn, William Miller, Joshua New and Paul Berdahl. (2012). "Understanding the Long-Term Effects of Environmental Exposure on Roof Reflectance in California." Construction and Building Materials 26(1):516-26.

Ferrari, C., G. Santunione, A. Libbra, A. Muscio, E. Sgarbi, C. Siligardi and G. S. Barozzi. (2015). "Review on the Influence

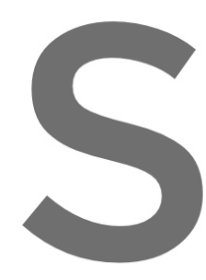
of Biological Deterioration International Journal of Des

Gaylarde, C. C. C., L. H. G. I . ( Paint Films - A Review.

Gaylarde, Christine C. and Peter Exteriors of Buildings in

Mastrapostoli, Elena, Matthaios (2016). "On the Ageing of Cool Roofs: Measure of the
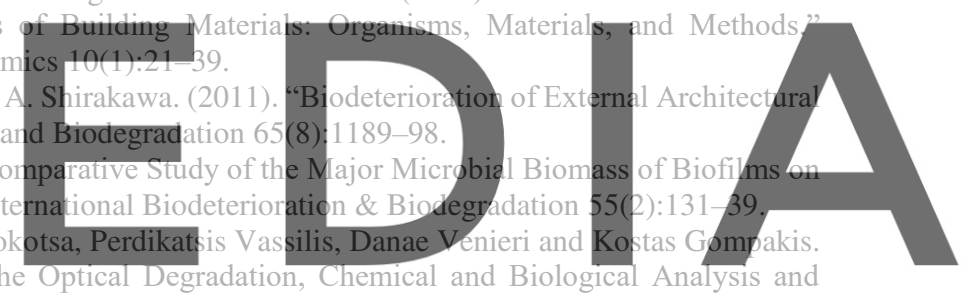
Assessment of the Energy Impact." Energy and Buildings 114:191-99.

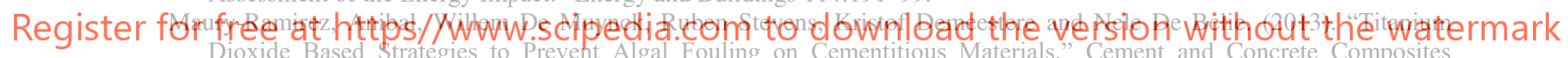
36(0):93-100.

Ortega-Calvo, J. J., X. Ariño, M. Hernandez-Marine and C. Saiz-Jimenez. (1995). "Factors Affecting the Weathering and Colonization of Monuments by Phototrophic Microorganisms." Science of The Total Environment 167(1-3):329-41.

Standard Test method for Solar Absorptance. ASTM E903, Performance, Measuring, Near Infrared Spectrophotom-, Air Mass, Zero Solar, Irradiance Tables, Sheet Materials, and Using Sunlight. (1996). "Standard Test Method for Solar Absorptance , Reflectance, and Transmittance of Materials Using Integrating Spheres 1.” 03:1-9.

Sleiman, Mohamad, Thomas W. Kirchstetter, Paul Berdahl, Haley E. Gilbert, Sarah Quelen, Lea Marlot, Chelsea V. Preble, Sharon Chen, Amandine Montalbano, Olivier Rosseler, Hashem Akbari, Ronnen Levinson and Hugo Destaillats. (2014). "Soiling of Building Envelope Surfaces and Its Effect on Solar Reflectance - Part II: Development of an Accelerated Aging Method for Roofing Materials." Solar Energy Materials and Solar Cells 122:271-81.

Welander, M., J. Persson, H. Asp and L. H. Zhu. (2014). "Evaluation of a New Vessel System Based on Temporary Immersion System for Micropropagation." Scientia Horticulturae. 